\title{
Pteridofitas indicadoras de alteración ambiental en el bosque templado de San Jerónimo Amanalco, Texcoco, México
}

Lucía Rodríguez Romero ${ }^{1}$, Leticia Pacheco ${ }^{2}$ \& José Alejandro Zavala Hurtado ${ }^{3}$

1. Doctorado en Ciencias Biológicas, Depto. de Biología, Universidad Autónoma Metropolitana-Iztapalapa, Apdo. Postal 55-535, 09340 México D.F., México; luciarr@prodigy.net.mx

2. Área de Botánica Estructural y Sistemática Vegetal, Depto. de Biología, Universidad Autónoma MetropolitanaIztapalapa, Apdo. Postal 55-535, 09340 México D.F., México; pacheco@xanum.uam.mx

3. Área de Ecología, Depto. de Biología, Universidad Autónoma Metropolitana-Iztapalapa, Apdo. Postal 55-535, 09340

México D.F., México; jazh@xanum.uam.mx

Recibido 26-II-2007. Corregido 08-XI-2007. Aceptado 04-IV-2008.

\begin{abstract}
Pteridophytes that indicate environmental alteration in the temperate forest of San Jerónimo Amanalco, Texcoco, México. Pteridophytes that indicate environmental alteration in the San Jerónimo Amanalco temperate forest, Texcoco, Mexico. The patterns of distribution of 26 pteridophyte species were studied as possible indicators of environmental alteration in the temperate forest of San Jerónimo Amanalco, Texcoco, State of Mexico. The presence and abundance of the pteridoflora was studied in relation to edaphic, topographic and vegetation variables in 100 sampling locations within an area of 494 hectares. The relationship between these variables was studied using Canonical Correspondence Analysis. Five landscapes were recognized in the study zone according to the degree of deterioration: severe erosion, erosion, mountain with moderate reversible deterioration, mountain with no evident deterioration, and canyon with no evident deterioration. Cheilanthes bonariensis and Pellaea ternifolia are indicators of environmental degradation. The taxa that only grow in landscapes without apparent alteration are Adiantum andicola, Adiantum poiretii, Argyrochosma incana, Asplenium blepharophorum, Dryopteris pseudo filix-mas, Equisetum hyemale and Pteris cretica. Rev. Biol. Trop. 56 (2): 641-656. Epub 2008 June 30.
\end{abstract}

Key words: ferns, Pteridophyta, Mexico, temperate forest, environmental deterioration.

El impacto ambiental de diversas actividades humanas se ha reflejado de manera directa sobre los recursos naturales, los diferentes paisajes y sobre la diversidad de flora y fauna a nivel mundial, nacional y regional, expresándose generalmente de manera negativa, ya que la actividad del hombre comúnmente produce alteraciones desfavorables y una degradación progresiva del ambiente.

La degradación como concepto implica la pérdida de las capacidades productivas del ambiente debido a procesos de erosión, salinización, pérdida de nutrientes y modificación de la estructura del suelo (Landa et al. 1997). Con el fin de precisar el término deterioro ambiental, Landa et al. (1997) lo definen como "una modificación del ambiente que implica una reducción o pérdida de sus cualidades físicas y biológicas, causadas por fenómenos naturales o por actividades humanas, representando finalmente una disminución de la disponibilidad de los bienes y servicios del ecosistema para las poblaciones humanas".

En 1978 los bosques del municipio de Texcoco contaban con una superficie de 225.89 $\mathrm{km}^{2}$, registrándose una reducción por desmonte y cambio de uso de suelo de $33.89 \mathrm{~km}^{2}$ (3 389 ha) en 1994, por lo que la superficie marcó un decremento del $8.10 \%$, siendo la velocidad de pérdida anual de bosque de $282.41 \mathrm{ha} /$ anuales (Escalona 2004).

En México, existen 1024 taxa de pteridofitas (Mickel y Smith 2004). Para el Estado de México, Tejero y Arreguín (2004) reconocieron 
248 taxa, de los cuales 60 se citan por primera vez. Además de la importante presencia de este grupo, su estudio como indicadoras de deterioro ambiental se apoya en la presencia de una serie de componentes básicos que las caracteriza, por ejemplo, poseen una gran capacidad de dispersión que se basa no sólo en la densidad de población en la periferia y en la movilidad de las esporas sino también, del número de dichos elementos que están en disposición de ser trasladados. El tipo de dispersión dominante es por el viento a larga distancia llamada dispersión anemócora (Tryon 1986, Barrington 1993). Algunas de las pteridofitas poseen gran capacidad de expansión considerándose como pródigas, oportunistas o pioneras, por ello son propias de las primeras etapas de la sucesión y su evolución ha sido tal, que su éxito consiste en la fácil colonización de ambientes vacíos adecuados como claros de bosques, selvas y zonas áridas además de áreas consideradas como "nuevas" que se abren de manera irregular y son de duración limitada (Zimmermann 1976, Strasburger et al. 1993). Finalmente, invaden ambientes perturbados como laderas taladas y bosques recientemente quemados, a causa de su producción de millones de esporas llevadas por el viento y por tener fuerte tolerancia al sol (Pérez-García et al. 1995, Riba 1998).

En este trabajo se realizaron estudios a nivel local en un bosque templado, con áreas que presentan elementos de paisaje muy heterogéneos, que se manifiestan en la diversidad y abundancia de la vegetación en cada uno de ellos. Además, su cercanía a importantes asentamientos humanos implica la presencia de diferentes condiciones de perturbación que son discernibles visualmente. Considerando que el disturbio de la vegetación provoca alteraciones en ciertas propiedades del suelo, aquí probamos la hipótesis de que ciertas especies de pteridofitas con diferente grado de especialización por condiciones edáficas particulares estarán asociadas con tipos específicos de perturbación, por lo que podrían considerase como especies indicadoras. Así, el objetivo de este estudio fue descubrir y describir el cambio de la abundancia y la diversidad de las pteridofitas, basado en factores edafológicos que pueden poner de manifiesto el grado de alteración ambiental local.

\section{MATERIALES Y MÉTODOS}

Área de estudio: el área de estudio se encuentra en la zona Oriente del Estado de México, en el municipio de Texcoco, el cual representa el $1.8 \%$ de la superficie del estado. Al norte del área de estudio se encuentra el municipio de Tepetlaoxtoc, al este los límites del Estado de Tlaxcala, al sur se perfila la zona montañosa, macizos que forman parte de la Sierra Nevada y al oeste las poblaciones de Santa María Tecuanulco y Santa Catarina del Monte (INEGI 2000, Cosío 2001). El paisaje que forma el área de estudio se distingue por una gran heterogeneidad en la vegetación, relieve y grado de deterioro, ocasionado o acelerado por la acción humana, lo que se refleja en gran diversidad de parches o elementos de paisaje. La localización del área de estudio es: (19 $30^{\prime}-19^{\circ} 31^{\prime}$ N, $\left.98^{\circ} 43^{\prime}-98^{\circ} 45^{\prime} \mathrm{W}\right)$, en San Jerónimo Amanalco.

Esta región forma parte de la provincia fisiográfica del Eje Neovolcánico, subprovincia de los lagos y volcanes de Anáhuac, en la topoforma de sierra. Ésta ocupa el $47.55 \%$ de la superficie del municipio (INEGI 2000, Cosío 2001). Geológicamente se caracteriza por suelos del Cenozoico, del periodo terciario, derivados de roca ígnea extrusiva en unidades litológicas de andesita y brecha sedimentaria (INEGI 2000). La mayor parte del suelo se encuentra formado por cambisol y pequeñas áreas por vertisoles y litosoles (INEGI 2000).

Los ríos que cruzan el municipio de Texcoco tienen su origen en la parte oriental de la Sierra Nevada, los cuales no son de caudal permanente y desembocan en su totalidad en lo que fuera el Lago de Texcoco. En el área de estudio, la corriente superficial corresponde al río Hueyapa (INEGI 2000). La mayoría de los manantiales observados en el área forman parte de la cañada denominada El Partidor. El clima es $\mathrm{C}(\mathrm{w} 2)$, que corresponde a un clima 
templado subhúmedo con lluvias en verano, de mayor humedad, y $\mathrm{C}(\mathrm{E})(\mathrm{w} 2)$ que corresponde a un clima semifrío subhúmedo con lluvias en verano, de mayor humedad (INEGI 2000, Cosío 2001). El sistema orográfico del área está constituido por cinco cerros que van de 2920 a $3040 \mathrm{~m}$. El gradiente altitudinal del área de estudio es de 2650 a $3040 \mathrm{~m}$. La vegetación de la zona consiste de bosque de encino y pino, pastizales inducidos y áreas de uso agrícola (INEGI 2000, Cosío 2001).

Muestreo: se ubicaron de manera sistemática 88 puntos de muestreo en una retícula con puntos de intersección separados cada $250 \mathrm{~m}$, más 12 sitios de muestreo elegidos subjetivamente por sus características particulares. En cada uno de los 88 puntos de intersección, más los 12 puntos adicionales, se colectó una muestra de un $\mathrm{kg}$ de suelo del horizonte A, hasta $10 \mathrm{~cm}$. de profundidad; se registraron en campo los datos de relieve, altitud, pendiente, orientación, descripción de materia orgánica y textura, tipo de vegetación y densidad de la misma, además del tipo de alteración evidente y grado de deterioro en su caso, estimados de manera visual.

Las muestras de suelo se colocaron en bolsas de polietileno para su transporte al laboratorio. El $\mathrm{pH}$ se midió con un potenciómetro digital marca Hanna Instruments HI 8314, en suspensión de suelo y agua destilada con relación suelo-agua de 1:2.5 para medir la concentración de iones hidrógeno solubles (Reyes Jaramillo 1996). El porcentaje de materia orgánica se determinó con la técnica desarrollada por Walkley y Black (Reyes Jaramillo 1996).

Además, en cada sitio de muestreo se marcaron cinco cuadros de $1 \times 1 \mathrm{~m}^{2}$ a lo largo de un transecto con dirección de norte a sur para el registro de la presencia de especies terrestres y helechos típicamente epífitos que se encontraron como formas terrestres. Sólo se tomaron en cuenta aquellos individuos en los que el rizoma quedó incluido dentro de cada cuadro.

En el área de estudio, se recolectaron en campo muestras de helechos y plantas afines durante dos años de recorrido conforme al método clásico señalado por Lorea y Riba (1990); posteriormente se procedió a identificarlas, utilizando claves taxonómicas especializadas (Smith 1981, Mickel y Beitel 1988, Moran y Riba 1995, Mickel y Smith 2004). El material de respaldo se depositó en el Herbario Metropolitano "Dr. Ramón Riba y Nava Esparza” (UAMIZ).

Banco de esporas: para tener una idea más clara acerca de la composición pteridológica del suelo del área de estudio por el depósito de esporas, se decidió hacer un estudio del número de gametofitos y esporofitos obtenidos de éstas. En laboratorio se separaron 50 gramos de cada muestra de suelo colectada, se tamizaron con una malla de $2 \mathrm{~mm}$ de apertura con el fin de separar gravas, restos vegetales y homogenizar el contenido de esporas. El suelo se depositó en un vaso térmico desechable de $125 \mathrm{ml}$ por muestra. Los 100 recipientes fueron puestos en charolas y cubiertos con bolsas de polietileno transparente, se regaron cada semana con agua potable hasta obtener esporofitos. Cada quince días se revisaron y contaron los gametofitos y/o esporofitos. Todas las muestras se mantuvieron en un estante, con lámparas de luz blanca de tubo fluorescente de $75 \mathrm{~W}$, con un fotoperiodo de 12 horas luz y 12 horas oscuridad a una temperatura de $24-28^{\circ} \mathrm{C}$.

Análisis numérico: De las 100 unidades de muestra, se separaron 30 por no haberse hallado en éstas individuos de pteridofitas y con las 70 muestras restantes se formaron tres matrices, una de variables físicas y edáficas y dos que corresponden a la abundancia y presencia/ausencia de las especies registradas en cada sitio de muestreo del área de estudio. Las dos matrices de especies fueron analizadas con un análisis multivariado de clasificación mediante el método de varianza mínima, a partir de matrices de distancias euclidianas (Gauch 1982).

La relación entre las variables ambientales y la abundancia o composición de especies se analizó mediante Análisis Canónico de Correspondencia (Gauch 1982). Este es un método de ordenación directa que genera 
gradientes que combinan linealmente variables ambientales y variables de especies. La diversidad de cada sitio se estimó utilizando el índice de Simpson (1- $\lambda$; Krebs 1985, Lande 1996). Los análisis multivariados fueron realizados por medio del Paquete Estadístico Multivariado (MVSP 3.2, Kovach 2003).

Con base en los grupos obtenidos del análisis de clasificación, se definieron paisajes correspondientes a tipos y grados de perturbación, considerando también propiedades de relieve, su relación directa con variables medioambientales y el grado observado de modificación del ecosistema.

\section{RESULTADOS}

Sitios con pteridofitas: los resultados corresponden a 70 unidades de muestreo en los que se registraron 1494 individuos de pteridofitas que pertenecen a 26 especies distribuidas en ocho familias de pteridofitas (Cuadro 1).

CUADRO 1

Especies de pteridofitas incluidas en el análisis

TABLE 1

Species of pteridophyte used in the analysis

Especie

Adiantum andicola Liebm.

Adiantum poiretii Wikstr.

Argyrochosma incana (C. Presl) Windham

Asplenium blepharophorum Bertol.

Asplenium monanthes L.

Athyrium arcuatum Liebm.

Cheilanthes arizonica (Maxon) Mickel

Cheilanthes bonariensis (Willd.) Proctor

Cheilanthes decurrens Mickel

Cheilanthes lendigera (Cav.) Sw.

Cheilanthes marginata Kunth

Cheilanthes myriophylla Desv.

Cystopteris fragilis (L.) Bernh.

Dryopteris pseudofilix-mas (Fée) Rothm.

Equisetum hyemale A. Braun ex Engelm. L. var. affine (Engelm.) A. A. Eaton

Pellaea cordifolia (Sessé et Moç) A. R. Sm.

Pellaea ternifolia (Cav.) Link

Phanerophlebia nobilis (Schltdl. et Cham.)

C. Presl var. nobilis

Pleopeltis conzattii (Weath.) R. M. Tryon et A. F. Tryon

Pleopeltis mexicana (Fée) Mickel et Beitel

Pleopeltis polylepis (Roem. ex Kunze) T. Moore var. interjecta (Weath.) E. A. Hooper

Polypodium thyssanolepis A. Braun ex Klotzsh

Pteris cretica L.

Selaginella sellowii Hieron.

Thelypteris linkiana (C. Presl) R. M. Tryon

Woodsia mollis (Kaulf.) J. Sm.
Ejemplar de respaldo

LRR-76, 167

LRR-82, 83, 86, 149, 158, 159, 160, 161, 162,

$163,164,165,166,168$

LRR-94, 142

LRR-100

LRR-75, 78, 80, 88, 102, 103, 145

LRR-132

LRR-90

LRR-91, 184, 186, 187, 188

LRR-169

LRR-77, 78,79, 85, 87, 89, 148, 151, 152, 153, 154, 155, 156

LRR-157

LRR-89a, 92

LRR-105, 108, 134, 141, 143, 144

LRR-113, 185

LRR-81, 140

LRR-93, 137, 138, 139

LRR-136,135

LRR-78, 101

LRR-173, 174, 176

LRR-111, 175, 177, 178

LRR-172

LRR-150

LRR-110

LRR-180, 182

LRR-99, 107, 117, 118, 119, 122, 123, 124, 125, 126, 127,

128,129

LRR-96, 170, 171, 179

LRR: Recolector Lucía Rodríguez Romero.

LRR: Collector Lucía Rodríguez Romero. 
La familia con mayor número de especies fue Pteridaceae (12), seguido de Polypodiaceae (4) y Aspleniaceae (3). Las especies con mayor frecuencia de presencia fueron Cheilanthes marginata en 45 sitios de muestra, $C$. lendigera presente en 43 sitios y $C$. bonariensis presente en 24 unidades de muestra. Durante sequía, en el sotobosque dominan helechos del género Pellaea y Cheilanthes, considerados como xerófilos. Cheilanthes marginata fue la especie que estuvo presente en la mayoría de los sitios, ya que se encontró en 45 de los 70 que tienen por lo menos una pteridofita, sin embargo, la especie de mayor abundancia relativa, con 655 individuos en total en el área de estudio fue $C$. lendigera, la cual estuvo presente en 43 sitios; la mayoría de las veces relacionada con $C$. marginata.

El resumen del proceso de clasificación numérica de 70 sitios de muestra en función de 26 especies de pteridofitas y plantas afines registradas en el área de estudio, se observa en el dendrograma de presencia-ausencia (Fig. 1). En esta figura se puede apreciar la formación de dos grupos que a su vez se subdividen para formar cuatro grupos que se distinguen claramente a una distancia euclidiana al cuadrado de 8.2. Los resultados del Análisis Canónico de Correspondencias (Fig. 2) indicaron que las variables ambientales más importantes son número de especies, cobertura, $\mathrm{pH}$ y altitud respectivamente, aunque el tipo de vegetación, cantidad de materia orgánica y número de esporofitos y gametofitos igualmente son importantes ya que determinan la presencia de algunas de las especies.

Con la clasificación numérica de 70 muestras en función de la abundancia se apreció la formación de un gran grupo que se puede subdividir para formar cinco subgrupos claramente distinguibles a una distancia euclidiana al cuadrado de 772.7 .

Sitios sin pteridofitas: el grupo formado por 30 muestras donde no se encontraron pteridofitas se caracterizó por la baja presencia de cobertura vegetal, hasta considerarla en algunos sitios como casi ausente a interrumpida, la vegetación primaria estuvo muy alterada, la vegetación secundaria formada por ruderales de tipo agrícola, erosión y reforestación con eucaliptos, $\mathrm{pH}$ ligeramente ácido a ligeramente alcalino y bajo contenido de materia orgánica.

Integración: con base en los análisis anteriores, se logró apreciar que la separación entre grupos y la relación de éstos con su respectiva correspondencia entre pteridofitas y variables ambientales, es positivamente distinguible cuando se considera la presencia/ausencia y en cambio, no resulta fácil visualizar la clasificación con base en la abundancia porque existen grupos cuyas variables no muestran un patrón general y parecen estar situadas en campo, en una fase de transición o de alteración intermedia. En términos generales, es posible considerar que el análisis que nos da más información sobre especies indicadoras es el de presencia/ausencia y que una vez determinadas entonces el análisis de abundancia se hace importante. Por lo anterior, se decidió determinar para el área de estudio cuatro grupos claramente distinguibles tanto por sus características ambientales como por la presencia de helechos, los que a su vez, al ser exclusivos tanto al estar presentes como por estar ausentes nos pueden servir como indicadores de alteraciones ambientales (Cuadro 2). El reconocimiento anterior se vio reforzado por el crecimiento en laboratorio, de gametofitos y esporofitos en las muestra de suelo colectadas para este fin (Cuadro 3).

Así, tenemos que en San JerónimoAmanalco se observaron áreas discretas dispersas, que en conjunto formaron cuatro paisajes básicos de acuerdo al relieve y grado de alteración (Cuadro 4), y éstos a su vez se vieron reflejados primero en la presencia y después en la abundancia de algunas pteridofitas, los paisajes son: erosión, montaña con deterioro moderado reversible, montaña sin deterioro aparente y cañada sin deterioro aparente (Fig. 2).

Erosión (Grupo 1, Fig. 1 y 2): se registraron 21 unidades de muestreo en áreas abiertas que fueron de cultivo pero que han sido abandonadas, dejando la superficie del terreno 


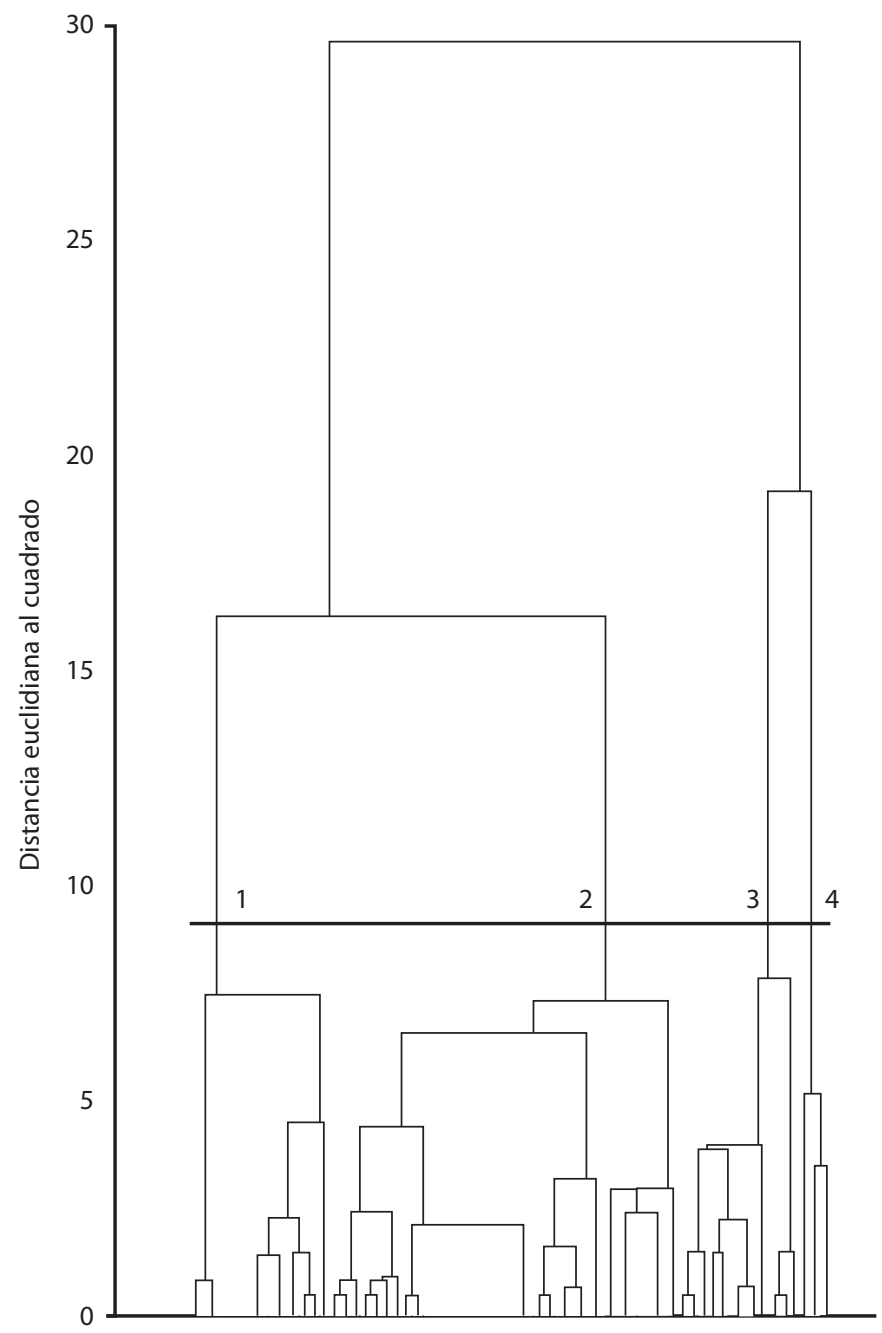

Fig. 1. Dendrograma con la clasificación de 70 sitios de muestreo con base en la presencia-ausencia de 26 especies de pteridofitas en el área de estudio. Los números indican los cuatro grupos considerados.

Fig. 1. Dendrogram of the classification of 70 sampling places on the basis of the presence-absence of 26 pteridophyte species in the study area. The numbers show the four groups considered.

indefensa ante la acción del agua en temporada de lluvias, provocando disminución drástica de la cubierta vegetal, arrastre de partículas de suelo, coladas de lodo, deslizamientos y formación de cárcavas. El cambio de uso del suelo forestal en zonas agrícolas o minas de arena, sobre todo en pendientes escarpadas o laderas, transforman el paisaje destruyendo el bosque y facilitando la erosión, de manera que el impacto ambiental es directo, severo y en ocasiones irreversible porque el efecto que produce dificulta la posibilidad de que, utilizando medidas correctoras, se pueda llegar al estado inicial de suelo de vocación forestal.

El suelo se caracterizó por bajo contenido de materia orgánica $(<3.5 \%)$, $\mathrm{pH}$ que tiende a variar en relación positiva con el grado de deterioro, de ligeramente ácido hasta 


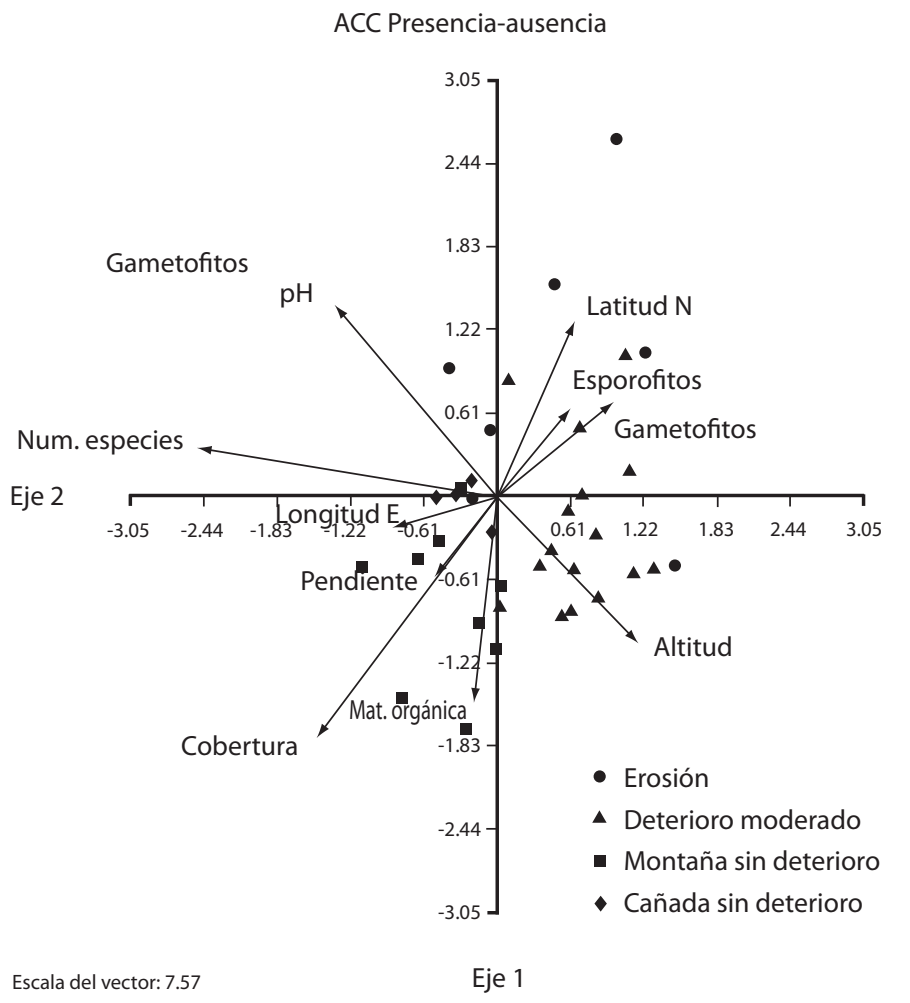

Fig. 2. Gráfico compuesto de la ordenación simultánea de 70 muestras de vegetación en función de la presencia-ausencia de 26 especies de pteridofitas y 10 variables ambientales en el área de estudio. Los vectores indican la importancia (longitud de la flecha) y sentido de la variación (dirección de la flecha) de las diez variables ambientales más importantes. Los números y símbolos indican los cuatro grupos considerados, relacionados con los paisajes observados de acuerdo con el relieve y grado de alteración.

Fig. 2. Graphic compound of the simultaneous arrangement of 70 vegetation samples in function of presence -absence of 26 pteridophyte species and 10 environmental variables in the studio area. The vectors show the importance (length of the arrow) and variation direction (direction of arrow) of the ten more important environmental variables. The symbols and numbers show the four groups considered, related with the observed landscape according with the relief and deterioration degree.

ligeramente alcalino. En paisajes erosionadas la riqueza se constriñe a tres especies, Cheilanthes marginata, presente en todos los grupos, disminuye su abundancia en estas áreas; sin embargo, C. bonariensis y Pellaea ternifolia son comunes, además de que son estos ambientes los que favorecen el aumento de su abundancia relativa.

De igual forma, en las muestras del banco de esporas individuos de los géneros Cheilanthes y Pellaea, fueron los primeros en germinar, después de catorce días de haber sido sembrados; formándose la primera hoja del esporofito entre 90 y 120 días después de la siembra.

Montaña con deterioro moderado reversible (Grupo 2, Fig. 1 y 2): Se identifica por 35 sitios de muestra que pueden ser considerados como de impacto ambiental moderado, porque no precisan de prácticas correctoras intensivas, como es el caso de la tala de árboles en zona boscosa, para autoconsumo; pero a su vez, esta misma práctica puede ser catalogada 
CUADRO 2

Resumen del arreglo por grupo de variables ambientales

TABLE 2

Abstract of the arrangement by group of registered variables

$\begin{array}{cccccccccc}\text { Grupo } & \text { Sitios } & \text { Taxa } & \text { Indice diversidad } & \text { Num. sp. } & \text { Indiv. } & \mathrm{pH} & \text { Mat. org. } & \text { Gam. } & \text { Esp. } \\ 1 & 21 & 8 & 0.13 & 1.43 & 85 & 6.40 & 2.80 & 45.4 & 20.10 \\ 2 & 35 & 14 & 0.66 & 3.18 & 843 & 5.60 & 4.30 & 46.4 & 19.97 \\ 3 & 11 & 14 & 0.63 & 4.45 & 360 & 6.27 & 3.68 & 9.30 & 2.20 \\ 4 & 3 & 22 & 0.94 & 15.67 & 260 & 5.80 & 3.59 & 12.7 & 6.00 \\ \text { ES } & 30 & 0 & 0 & 0 & 0 & 6.41 & 2.14 & 10.9 & 4.27\end{array}$

ES: erosión severa. Taxa: número máximo de especies registrado. Índice diversidad: Índice de Simpson promedio. Num sp: número promedio de especies de pteridofitas presentes en cada unidad de muestreo. pH: promedio por grupo de $\mathrm{pH}$, Mat org: porcentaje de materia orgánica, Gam: número de gametofitos Esp: número de esporofitos registrados en el banco de esporas.

Variables registradas en 100 sitios de muestreo en San Jerónimo Amanalco, Texcoco, Estado de México.

ES: strict erosion. Taxa: maximum number of registered species. Diversity Index: Average Simpson Index. Num sp: Pteridophyte species average number in each sample unit. $\mathbf{p H}$ : Average by group of $\mathrm{pH}$, Mat org: percentage of organic material, Gam: number of gametophytes Esp: sporophytes reported in the soil spore bank.

Registered variables in 100 sampling places in San Jeronimo Amanalco, Texcoco, Estado de Mexico.

como impacto directo, aunque sus efectos no tengan incidencia inmediata en el medio. Los parches localizados con evidencias claras de haber sufrido incendios pueden caracterizarse como impactos reversibles en la medida que se apliquen acciones correctoras y a su vez, de tipo periódico por ser fenómenos "normales" en sequía de cada año.

De acuerdo al tipo de vegetación, se observaron paisajes de vegetación primaria de bosques de encino, pino, oyamel y cedro, ubicados en relieve montañoso con pendiente moderadamente escarpada. Asimismo, presentan parches afectados por incendios forestales y tala de árboles, causas que aceleran el proceso erosivo en temporada de lluvias; en resumen, son bosques que poseen vegetación original y que están seriamente amenazados. En su caso, la vegetación primaria ha sido sustituida en pequeños parches por áreas de cultivo, incendios forestales, reforestación con eucaliptos y terrenos deforestados, ocasionando la disminución de materia orgánica. Sin embargo, cabe destacar que la cantidad de materia orgánica no es una variable que, en este tipo de paisaje, tenga relación con el tipo de deterioro o con variables ambientales o edáficas; en cambio, el $\mathrm{pH}$ tiene valores con márgenes restringidos ya que varían de ácido a ligeramente ácido (4.9-6.9), en relación positiva con el grado de deterioro. En cuanto a la presencia de pteridofitas, dos especies son comunes en estos paisajes, Cheilanthes marginata y Cheilanthes lendigera, pero C. marginata tiende a incrementar su abundancia relativa en fragmentos abiertos que presentan algún tipo de deterioro. C. bonariensis y Pellaea ternifolia se ubicaron en sitios con deterioro evidente, o en sitios en donde se relacionó el porcentaje de materia orgánica bajo con $\mathrm{pH}$ ligeramente ácido a ligeramente alcalino (6-8).

Cheilanthes bonariensis y Pellaea ternifolia, no sólo se observaron en los sitios de mayor deterioro sino que también formaron parte de paisajes modificados drásticamente por desmonte, incendio y áreas de cultivo abandonadas. Además, dichas especies no son comunes en áreas en la que existe vegetación 
CUADRO 3 / TABLE 3

Gametofitos y esporofitos por muestra de suelo / Gametophytes and sporophytes by soil sample

\begin{tabular}{|c|c|c|c|c|c|c|c|}
\hline Grupo & Muestra & $\begin{array}{l}\text { Número de } \\
\text { Gametofitos }\end{array}$ & $\begin{array}{l}\text { Número de } \\
\text { Esporofitos }\end{array}$ & $\begin{array}{c}\text { Germinación } \\
35 \text { días }\end{array}$ & $\begin{array}{c}\text { Gametofitos } \\
\text { 155días }\end{array}$ & $\begin{array}{c}\text { Esporofito } \\
155 \text { días }\end{array}$ & $\begin{array}{l}\text { Esporofit } \\
300 \text { días }\end{array}$ \\
\hline 1 & A1 & 0 & 0 & 0 & 0 & 0 & 0 \\
\hline E S & $\mathrm{A} 2$ & 8 & 3 & 4 & 8 & 3 & 0 \\
\hline E S & A3 & 9 & 0 & 3 & 9 & 0 & 0 \\
\hline E S & A4 & 0 & 0 & 0 & 0 & 0 & 0 \\
\hline E S & A5 & 4 & 0 & 0 & 4 & 0 & 0 \\
\hline 1 & A6 & 37 & 24 & 41 & 37 & 24 & 0 \\
\hline 2 & A6a & 27 & 2 & 5 & 27 & 2 & 0 \\
\hline E S & A7 & 2 & 1 & 3 & 2 & 1 & 1 \\
\hline 2 & A8 & 206 & 56 & 79 & 206 & 56 & 0 \\
\hline 3 & B1 & 1 & 1 & 5 & 1 & 1 & 0 \\
\hline 1 & B2 & 30 & 6 & 33 & 30 & 6 & 0 \\
\hline 1 & B3 & 11 & 7 & 7 & 11 & 7 & 0 \\
\hline E S & B4 & 1 & 0 & 1 & 1 & 0 & 0 \\
\hline E S & B5 & 0 & 0 & 0 & 0 & 0 & 0 \\
\hline 2 & B5a & 0 & 0 & 0 & 0 & 0 & 0 \\
\hline 2 & B6 & 50 & 21 & 0 & 50 & 21 & 1 \\
\hline 1 & B6a & 112 & 45 & 60 & 112 & 45 & 1 \\
\hline 2 & $\mathrm{~B} 6 \mathrm{~b}$ & 2 & 2 & 7 & 2 & 2 & 1 \\
\hline 1 & B7 & 13 & 6 & 15 & 13 & 6 & 1 \\
\hline 1 & B8 & 221 & 29 & 218 & 221 & 29 & 0 \\
\hline 1 & $\mathrm{C} 1$ & 19 & 7 & 11 & 19 & 7 & 0 \\
\hline 2 & $\mathrm{C} 2$ & 16 & 4 & 8 & 16 & 4 & 0 \\
\hline 2 & C3 & 13 & 4 & 12 & 13 & 4 & 0 \\
\hline E S & $\mathrm{C} 4$ & 2 & 0 & 0 & 2 & 0 & 0 \\
\hline 2 & C5 & 17 & 6 & 17 & 17 & 6 & 0 \\
\hline 2 & C6 & 60 & 12 & 12 & 60 & 12 & 1 \\
\hline 3 & C6a & 17 & 6 & 9 & 17 & 6 & 0 \\
\hline 1 & $\mathrm{C} 7$ & 6 & 1 & 11 & 6 & 1 & 1 \\
\hline 3 & $\mathrm{C} 7 \mathrm{~b}$ & 1 & 0 & 0 & 1 & 0 & 0 \\
\hline 1 & $\mathrm{C} 8$ & 20 & 12 & 11 & 20 & 12 & 0 \\
\hline E S & D1 & 12 & 2 & 4 & 12 & 2 & 1 \\
\hline 2 & D2 & 13 & 4 & 8 & 13 & 4 & 0 \\
\hline 2 & D3 & 15 & 4 & 8 & 15 & 4 & 0 \\
\hline 2 & D4 & 7 & 1 & 3 & 7 & 1 & 0 \\
\hline 3 & D5 & 43 & 4 & 32 & 43 & 4 & 0 \\
\hline 2 & D6 & 42 & 19 & 69 & 42 & 19 & 1 \\
\hline 1 & D7 & 50 & 25 & 34 & 50 & 25 & 1 \\
\hline 3 & D7a & 11 & 1 & 0 & 11 & 1 & 0 \\
\hline E S & D8 & 3 & 0 & 1 & 3 & 0 & 0 \\
\hline E S & E1 & 6 & 1 & 8 & 6 & 1 & 0 \\
\hline
\end{tabular}


CUADRO 3 / TABLE 3 (Continuación / Continued)

Gametofitos y esporofitos por muestra de suelo / Gametophytes and sporophytes by soil sample

\begin{tabular}{|c|c|c|c|c|c|c|c|}
\hline Grupo & Muestra & $\begin{array}{l}\text { Número de } \\
\text { Gametofitos }\end{array}$ & $\begin{array}{l}\text { Número de } \\
\text { Esporofitos }\end{array}$ & $\begin{array}{l}\text { Germinación } \\
35 \text { días }\end{array}$ & $\begin{array}{l}\text { Gametofitos } \\
\text { 155días }\end{array}$ & $\begin{array}{c}\text { Esporofito } \\
155 \text { días }\end{array}$ & $\begin{array}{c}\text { Esporofito } \\
300 \text { días }\end{array}$ \\
\hline 2 & E2 & 16 & 8 & 12 & 12 & 0 & 1 \\
\hline 3 & E2a & 1 & 0 & 0 & 0 & 0 & 0 \\
\hline 2 & E3 & 20 & 6 & 11 & 20 & 6 & 1 \\
\hline E S & E4 & 55 & 9 & 38 & 55 & 9 & 0 \\
\hline 2 & E5 & 72 & 19 & 126 & 72 & 19 & 0 \\
\hline 2 & E6 & 39 & 20 & 42 & 39 & 20 & 0 \\
\hline 1 & E7 & 14 & 6 & 18 & 14 & 6 & 0 \\
\hline 1 & E8 & 111 & 65 & 74 & 111 & 65 & 1 \\
\hline 3 & F1 & 3 & 1 & 4 & 3 & 1 & 0 \\
\hline E S & $\mathrm{F} 2$ & 16 & 1 & 15 & 16 & 1 & 0 \\
\hline 1 & F3 & 9 & 2 & 0 & 9 & 2 & 0 \\
\hline 3 & F4 & 0 & 0 & 0 & 0 & 0 & 0 \\
\hline 2 & F5 & 0 & 0 & 0 & 0 & 0 & 0 \\
\hline 2 & F6 & 48 & 26 & 65 & 48 & 26 & 0 \\
\hline 2 & F7 & 19 & 5 & 10 & 19 & 5 & 0 \\
\hline 2 & F8 & 4 & 2 & 5 & 4 & 2 & 0 \\
\hline E S & G1 & 2 & 0 & 0 & 2 & 0 & 0 \\
\hline E S & G2 & 26 & 10 & 14 & 26 & 10 & 1 \\
\hline 2 & G3 & 8 & 4 & 12 & 8 & 4 & 0 \\
\hline 2 & G4 & 7 & 3 & 17 & 7 & 3 & 0 \\
\hline 2 & G5 & 34 & 6 & 45 & 34 & 6 & 0 \\
\hline 2 & G6 & 98 & 8 & 45 & 98 & 8 & 1 \\
\hline 2 & G7 & 100 & 90 & 103 & 100 & 90 & 0 \\
\hline E S & G8 & 0 & 0 & 0 & 0 & 0 & 0 \\
\hline E S & H1 & 8 & 2 & 9 & 8 & 2 & 0 \\
\hline E S & $\mathrm{H} 2$ & 6 & 3 & 18 & 6 & 3 & 0 \\
\hline 2 & H3 & 1 & 0 & 0 & 1 & 0 & 0 \\
\hline 2 & $\mathrm{H} 4$ & 61 & 4 & 10 & 61 & 4 & 0 \\
\hline 2 & H5 & 13 & 5 & 14 & 13 & 5 & 0 \\
\hline 2 & H6 & 57 & 28 & 49 & 57 & 28 & 1 \\
\hline 1 & $\mathrm{H} 7$ & 32 & 1 & 10 & 32 & 1 & 0 \\
\hline 2 & H8 & 100 & 95 & 83 & 100 & 95 & 0 \\
\hline 3 & I1 & 7 & 0 & 7 & 7 & 0 & 0 \\
\hline 3 & I2 & 13 & 8 & 11 & 13 & 8 & 1 \\
\hline 1 & I3 & 1 & 0 & 0 & 1 & 0 & 0 \\
\hline 2 & I4 & 105 & 70 & 114 & 105 & 70 & 0 \\
\hline E S & I5 & 71 & 53 & 94 & 71 & 53 & 0 \\
\hline 1 & I6 & 14 & 12 & 6 & 14 & 12 & 1 \\
\hline 1 & I7 & 46 & 5 & 15 & 46 & 5 & 0 \\
\hline 1 & I8 & 20 & 7 & 4 & 20 & 7 & 0 \\
\hline
\end{tabular}


CUADRO 3 / TABLE 3 (Continuación / Continued)

Gametofitos y esporofitos por muestra de suelo / Gametophytes and sporophytes by soil sample

$\begin{array}{cccccccc}\text { Grupo } & \text { Muestra } & \begin{array}{c}\text { Número de } \\ \text { Gametofitos }\end{array} & \begin{array}{c}\text { Número de } \\ \text { Esporofitos }\end{array} & \begin{array}{c}\text { Germinación } \\ 35 \text { días }\end{array} & \begin{array}{c}\text { Gametofitos } \\ 155 \text { días }\end{array} & \begin{array}{c}\text { Esporofito } \\ 155 \text { días }\end{array} & \begin{array}{c}\text { Esporofito } \\ 300 \text { días }\end{array} \\ \text { E S } & \text { J1 } & 11 & 2 & 16 & 11 & 2 & 0 \\ \text { E S } & \text { J2 } & 4 & 4 & 3 & 4 & 4 & 1 \\ 4 & \text { J2a } & 0 & 0 & 0 & 0 & 0 & 0 \\ 2 & \text { J3 } & 40 & 15 & 7 & 40 & 15 & 1 \\ 4 & \text { J3a } & 30 & 28 & 30 & 30 & 18 & 0 \\ \text { E S } & \text { J4 } & 2 & 2 & 7 & 2 & 2 & 1 \\ 4 & \text { J4a } & 6 & 0 & 9 & 6 & 0 & 0 \\ 2 & \text { J5 } & 240 & 130 & 144 & 240 & 130 & 0 \\ 2 & \text { J6 } & 22 & 4 & 20 & 22 & 4 & 0 \\ \text { E S } & \text { J7 } & 11 & 7 & 11 & 11 & 7 & 0 \\ 1 & \text { J8 } & 167 & 155 & 214 & 167 & 155 & 0 \\ \text { E S } & \text { K1 } & 7 & 2 & 13 & 7 & 2 & 1 \\ \text { E S } & \text { K2 } & 7 & 4 & 4 & 7 & 4 & 0 \\ 3 & \text { K3 } & 5 & 3 & 27 & 5 & 3 & 0 \\ \text { E S } & \text { K4 } & 23 & 5 & 9 & 23 & 5 & 0 \\ \text { E S } & \text { K5 } & 13 & 6 & 17 & 13 & 6 & 0 \\ \text { E S } & \text { K6 } & 4 & 3 & 2 & 4 & 3 & 0 \\ 1 & \text { K7 } & 20 & 7 & 14 & 20 & 7 & 0 \\ \text { E S } & \text { K8a } & 51 & 10 & 9 & 51 & 10 & 1 \\ \text { E S } & \text { K8b } & 14 & 5 & 5 & 14 & 5 & 0 \\ & \text { Total } & & 1494 & 2400 & 3043 & 1282 & 23 \\ & \text { individuos } & & & & & & \\ & & & & & & & \\ \end{array}$

Número de gametofitos y número de esporofitos se refiere al número registrado a los 170 días después de la siembra. Número de gametofitos incluye: neutros, con gametangios y con las primeras hojas del esporofito. El número de esporofitos incluye: los que aún están unidos al gametofito, los de origen sexual, y los apogámicos. Los esporofitos de 300 días son de talla mayor a $20 \mathrm{~cm}$.

Number of gametophytes and sporophytes refers to the number reported 170 days after sowing. The number of gametophytes includes neuter gametophytes, gametophytes with gametangia and gametophytes with the first sporophyte leaves. The number of the sporophytes includes those sporophytes that are still united with gametophytes, those with sexual origin and the apogamic. The sporophytes of 300 days are bigger than $20 \mathrm{~cm}$.

primaria sin alteraciones evidentes, por lo tanto únicamente se observan en los bosques que han sufrido algún tipo de impacto ambiental negativo y por ende, se puede decir que las tres especies son indicadoras de alteración ambiental negativa.

El tipo de perturbación o alteración observado en el grupo fue diverso, ya que contó con paisajes de erosión moderada, tala de árboles para autoconsumo, áreas de cultivo, reforestación con eucaliptos y una franja que sufrió incendios forestales. Probablemente es esta diversidad de paisajes la razón por la que no se puede señalar, a diferencia del grupo anterior, un patrón de crecimiento común en los helechos. La germinación de esporas en general destacó por su precocidad en la formación del esporofito para Cheilanthes, formándose 
CUADRO 4

Características por sitio de muestreo de cada grupo obtenido con base en la presencia-ausencia de especies de pteridofitas

TABLE 4

Characteristics by sample place of each group obtained using presence-absence of pteridophyte species

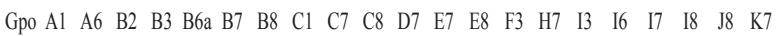

$\begin{array}{llllllllllllllllllllllll}\text { Num Sp's } & 1 \mathrm{P} & 1 & 3 & 1 & 3 & 2 & 1 & 1 & 1 & 6 & 1 & 1 & 1 & 1 & 1 & 1 & 2 & 1 & 2 & 2 & 1 & 1\end{array}$

$\begin{array}{llllllllllllllllllllllll}\text { Cob } & 1 \mathrm{P} & 3 & 4 & 4 & 6 & 5 & 3 & 2 & 2 & 4 & 1 & 3 & 5 & 2 & 4 & 3 & 4 & 2 & 1 & 1 & 1 & 2\end{array}$

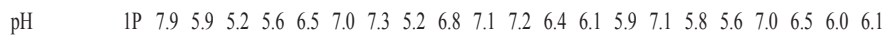

$\begin{array}{lllllllllllllllllllllllllll}\text { Mat org } \quad 1 P & 0.9 & 4.4 & 2.1 & 1.5 & 6.8 & 3.8 & 2.1 & 8.4 & 5.9 & 1.0 & 0.3 & 8.1 & 1.8 & 2.7 & 1.0 & 2.8 & 0.4 & 0.4 & 0.7 & 2.2 & 1.6\end{array}$

$\begin{array}{lllllllllllllllllllllll}\text { Rel } & 1 \mathrm{P} & 3 & 2 & 3 & 3 & 1 & 1 & 2 & 3 & 1 & 2 & 2 & 2 & 2 & 3 & 2 & 3 & 2 & 1 & 2 & 2 & 3\end{array}$

Gpo A6a A8 B5a B6 B6b C2 23 C5

$\begin{array}{llllllllllllllllllllllllllllllllllllll}\text { Num Sp's } & 2 \mathrm{P} & 3 & 7 & 2 & 4 & 4 & 3 & 2 & 2 & 2 & 5 & 2 & 2 & 6 & 2 & 5 & 3 & 3 & 3 & 2 & 3 & 2 & 2 & 2 & 4 & 2 & 3 & 3 & 4 & 2 & 1 & 7 & 4 & 4 & 5 & 2\end{array}$

$\begin{array}{lllllllllllllllllllllllllllllllllllllllllllllll}\mathrm{Cob} & 2 \mathrm{P} & 1 & 5 & 2 & 5 & 3 & 5 & 3 & 6 & 4 & 4 & 5 & 5 & 5 & 6 & 5 & 5 & 3 & 4 & 5 & 5 & 4 & 4 & 5 & 4 & 6 & 3 & 4 & 5 & 5 & 3 & 4 & 3 & 4 & 5 & 4\end{array}$

$\begin{array}{lllllllllllllllllllllllllllllllllllllll} & \mathrm{pH} & 2 \mathrm{P} & 6.1 & 6.4 & 7.0 & 6.1 & 6.3 & 5.2 & 5.1 & 5.4 & 5.1 & 6.4 & 4.9 & 5.5 & 5.2 & 5.8 & 5.4 & 5.1 & 5.3 & 6.0 & 5.0 & 5.6 & 5.8 & 5.5 & 4.9 & 4.7 & 5.0 & 7.7 & 5.2 & 5.3 & 5.2 & 6.0 & 6.2 & 6.1 & 5.6 & 5.6 & 5.5\end{array}$

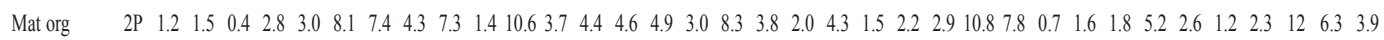

$\begin{array}{llllllllllllllllllllllllllllllllllllll}\text { Rel } & 2 \mathrm{P} & 2 & 1 & 2 & 3 & 1 & 3 & 3 & 3 & 3 & 2 & 3 & 3 & 1 & 2 & 3 & 3 & 3 & 3 & 3 & 3 & 1 & 3 & 3 & 3 & 3 & 1 & 3 & 3 & 3 & 2 & 2 & 2 & 3 & 1 & 1\end{array}$

Gpo B1 C6a C7b D5 D7a E2a F1 F4 I1 I2 K3

$\begin{array}{lllllllllllll}\text { Num } S p ' s & 3 P & 5 & 7 & 7 & 4 & 3 & 8 & 1 & 4 & 1 & 2 & 7\end{array}$

$\begin{array}{lllllllllllll}\mathrm{Cob} & 3 \mathrm{P} & 6 & 4 & 5 & 5 & 5 & 5 & 5 & 5 & 6 & 6 & 5\end{array}$

$\begin{array}{lllllllllllll}\mathrm{pH} & \text { 3P } & 5.0 & 5.8 & 7.9 & 5.4 & 7.2 & 7.7 & 6.3 & 6 & 5.8 & 6.2 & 5.68\end{array}$

$\begin{array}{llllllllllll}\text { Mat org } \quad 3 P & 8.0 & 5.4 & 1.7 & 5.1 & 4 & 1.8 & 1.1 & 3.6 & 1.8 & 2.8 & 4.59\end{array}$

$\begin{array}{lllllllllllll}\text { Rel } & 3 \mathrm{P} & 3 & 1 & 1 & 3 & 1 & 1 & 3 & 3 & 2 & 3 & 1\end{array}$

Gpo J2a J3a J4a

$\begin{array}{lllll}\text { Num. Sp's } \quad 4 P & 18 & 14 & 15\end{array}$

$\begin{array}{lllll}\mathrm{Cob} & 4 \mathrm{P} & 5 & 5 & 5\end{array}$

$\begin{array}{lllll}\mathrm{pH} & 4 \mathrm{P} & 5.6 & 5.0 & 6.7\end{array}$

$\begin{array}{lllll}\text { Mat org } \quad \text { 4P } & 6.2 & 2.0 & 2.7\end{array}$

$\begin{array}{lllll}\text { Rel } & 4 \mathrm{P} & 1 & 1 & 1\end{array}$

Num sp's: número de especies. Cob, cobertura vegetal:1, casi ausente; 2, rala; 3, dispersa; 4, interrumpida; 5, densa; 6, continua. Mat org: porcentaje de materia orgánica. Rel, tipo de relieve: 1, cañada 2, lomerío; 3, montañoso.

Pteridofitas registradas en el área de estudio.

Num sp's: species number. Cob, plant cover: 1, almost absent; 2, sparse; 3, scattered; 4, interrupted; 5, dense; 6, continuous. Mat org: organic material percentage. Rel, relief type: 1, ravine; 2, hill; 3, mountainous.

Pteridophyte species reported in the study area.

antes de 155 días; en cambio, individuos de los géneros Asplenium, Cystopteris y Thelypteris formaron esporofitos entre 155 a 300 días después de sembrados.

Montaña sin deterioro aparente (Grupo 3, Fig. 1 y 2): El bosque templado incluye bosques de encino, pino, oyamel o cultivados (Rzedowski 1978); en el área de estudio, 11 unidades de muestra, los bosques se caracterizan por ser caducifolios en sequía, de noviembre a marzo generalmente. Tienen alto contenido de materia orgánica y perfil del suelo profundo, lo que significa que cuentan con capas de la 
superficie hacia adentro de: hojarasca reciente, hojarasca en descomposición, restos orgánicos no identificables, hasta la capa externa del horizonte A que contiene suelo mineral enriquecido en materia orgánica y una zona de eluviación de humus y arcilla (Bennet y Humphries 1981), en sitios con éstas características se llegó a medir hasta cuarenta centímetros a partir de hojarasca reciente hasta llegar al horizonte A en julio. Los bosques con vegetación primaria se ubicaron en relieve de montaña, siendo estos los que tienen las condiciones propicias para el establecimiento de poblaciones de helechos. Es de llamar la atención la abundancia de algunas especies como Adiantum poiretii, Asplenium monanthes, Cheilanthes myriophylla, Pellaea cordifolia y Woodsia mollis; pero sobre todos éstos helechos, $C$. lendigera, presenta la mayor abundancia relativa del paisaje.

El paisaje representa la vegetación primaria clásica de bosque templado; por lo que la presencia constante de Adiantum poiretii, Asplenium monanthes, C. myriophylla, Pellaea cordifolia y Woodsia mollis, denota áreas que no han sufrido alteraciones o modificaciones graves que hayan impactado los componentes del medio.

Basándose en lo anterior, es posible suponer que los táxones de un bosque templado incluyen tanto elementos de flora xérica, como elementos de flora cálido-húmeda, además de elementos que requieren de alto contenido de materia orgánica; esto es porque en bosques templados, aproximadamente medio año es de sequía y el resto de mayor humedad por las lluvias. Lo anterior se refuerza si se toma en cuenta la presencia de Cheilanthes bonariensis, C. marginata, $C$. lendigera, $C$. decurrens, $C$. myriophylla, Pellaea cordifolia y P. ternifolia, considerados por Riba (1994) como helechos de zonas secas y semisecas del país.

En contraste, en algunos sitios del bosque templado se registraron Adiantum poiretii, Dryopteris pseudo filix-mas y Thelypteris linkiana, especies que sólo se observan en los sitios de mayor humedad.

El grupo de parches que no fueron afectados por perturbaciones evidentes, se caracterizó por la alta densidad de gametofitos obtenidos y porque la fase esporofítica se presentó después de 155 días de la siembra. Los géneros que germinaron son Adiantum, Asplenium, Cheilanthes, Cystopteris, Pellaea, Pleopeltis y Thelypteris.

Cañada sin deterioro aparente (Grupo 4, Fig. 1 y 2): El lugar propicio para el crecimiento de pteridofitas y plantas afines en bosques templados son las cañadas, porque es en ellas donde se depositan partículas de suelo y materia orgánica, existen buenas condiciones de drenaje y se mantiene la humedad durante sequía. En todas las cañadas, sobre todo en tres sitios de muestra localizados en El Partidor, se registró la mayor riqueza de especies, pero a su vez, la abundancia relativa disminuye, probablemente por la competencia que se establece por el espacio.

Adiantum andicola y Pellaea cordifolia no fueron abundantes, pero sí constantes en todos los sitios. Cabe resaltar que $A$. andicola únicamente fue observado en las cañadas. Las especies exclusivas de este grupo son $A$. andicola, Argyrochosma incana, Asplenium blepharophorum, Dryopteris pseudo filix-mas, Equisetum hyemale y Pteris cretica. La especie A. incana se observó exclusivamente en la cañada El Partidor, orientada geográficamente hacia el sur, lo que la ubica en el área de mayor humedad, en cambio, Dryopteris pseudo filixmas, Equisetum hyemale y Phanerophlebia nobilis sólo crecen en los márgenes del río.

Los helechos típicos de cañada: Adiantum poiretii, Argyrochosma incana, Asplenium blepharophorum, Pellaea cordifolia, Phanerophlebia nobilis, Pleopeltis mexicana, Thelypteris linkiana y Woodsia mollis tuvieron un ciclo de vida caracterizado por germinación de esporas entre catorce y veintitrés días. Después de cuarenta días el gametofito tuvo forma espatulada o cordiforme, presentándose la formación de los primeros esporofitos entre cien y ciento veinte días después de la siembra. Thelypteris siguió este mismo patrón, con la diferencia de que después que surgió la primera hoja del esporofito, su desarrollo fue el más 
acelerado, de tal manera que a los trescientos días de edad, alcanzaron una talla superior a $\operatorname{los} 20 \mathrm{~cm}$.

Este grupo de taxa se desarrolla en campo en sitios húmedos, aún en los meses secos, donde la cubierta vegetal impide radiación excesiva y exposición al viento; el pH del suelo así como la cantidad de nutrientes favorecen el establecimiento y crecimiento de gametofitos hasta formar el esporofito. De esta forma, se puede intuir que si el ambiente no sufre alteraciones importantes, de igual forma mantiene en equilibrio el tamaño de las poblaciones.

Erosión severa (desertificación; ausencia de pteridofitas): Conformado por 30 unidades de muestreo. Los paisajes observados con un proceso de erosión muy avanzado, al grado de tener cobertura vegetal rala, casi ausente o carecer de vegetación, tuvieron en común la relación negativa entre la cantidad de materia orgánica y el grado de acidez $(\mathrm{pH})$. Asimismo, la evidente ausencia de pteridoflora nos permite inferir lo avanzado del proceso de erosión hasta el punto de suponer la imposibilidad o dificultad de retornar a una recuperación de la vegetación primaria. No obstante el grado de deterioro, en 27 de los 30 sitios en los que no hubo presencia de pteridofitas, si hubo germinación en las muestras de suelo empleadas para el banco de esporas, sin embargo, la densidad de gametofitos fue evidentemente menor en relación a los sitios en los que si hubo presencia de pteridofitas.

Finalmente, en paisajes en los que la erosión es patente, la densidad de esporas que germinaron en las muestras de suelo es mayor que en el resto de paisajes y en cambio, en donde no existe alteración evidente el número de gametofitos y esporofitos fue menor. Cheilanthes germinó en mayor número en comparación con el resto de los géneros estudiados, de tal manera que los espacios abiertos, erosionados, resultado de cambio de uso de suelo, campos de cultivo o ruderales favorecen su crecimiento porque las esporas resisten mayor luminosidad, menor humedad y menor cantidad de nutrientes en comparación de aquellas especies que requieren de condiciones muy particulares para propiciar la germinación de sus esporas.

Las condiciones edáficas y ambientales que para la mayoría de los helechos resultan desfavorables, en especies como Cheilanthes bonariensis y $C$. marginata resultan benéficas gracias a algunas de sus peculiaridades, las esporas esparcidas por el viento son viables aun en condiciones de baja humedad y mayor exposición a los rayos del sol, son de germinación y crecimiento rápido, características apropiadas para el ambiente templado en donde la temporada de sequía es pronunciada y por si lo anterior no fuera suficiente, los esporofitos adultos pueden sobrevivir en condiciones de sequía extrema y rehidratarse rápidamente en la temporada de lluvias, condición propicia para invadir suelos delgados, grietas y cárcavas producto del proceso erosivo presente en la zona.

\section{CONCLUSIONES}

Los métodos de análisis multivariados utilizados (clasificación y ordenación directa) fueron adecuados para evaluar la relación de las variables medioambientales con los patrones de distribución de las especies y lograr la definición de paisajes.

San Jerónimo Amanalco, Texcoco, Estado de México, mostró 4 tipos de paisajes de acuerdo a sus características ambientales, grado de deterioro y presencia de pteridoflora: erosión, montaña con deterioro moderado reversible, montaña sin deterioro aparente y cañada sin deterioro aparente, además de un paisaje denominado erosión severa por sus características edafo-ecológicas y por la ausencia de pteridofitas.

C. bonariensis y P. ternifolia son indicadoras de alteración y deterioro ambiental.

C. marginata es una especie que tiende a incrementar su abundancia relativa en áreas abiertas y con indicios de deterioro ambiental.

$C$. lendigera incrementa su abundancia relativa en paisajes de montaña sin alteración aparente.

Los táxones que únicamente crecen en paisajes sin alteración aparente son: $A$. andicola, A. poiretii, A. incana, A. blepharophorum, $D$. pseudo filix-mas, E. hyemale y $P$. cretica. 
D. pseudo filix-mas, E. hyemale y P. nobilis sólo crecen en los márgenes del río.

Áreas que han sido cultivadas por eucaliptos como estrategia para reducir la erosión, disminuye la diversidad y abundancia de especies de pteridofitas.

\section{AGRADECIMIENTOS}

Este trabajo formó parte de los estudios de Maestría en la Universidad Autónoma Metropolitana, Iztapalapa de la primera autora, bajo la tutoría de los segundos. Se agradece la beca del Consejo Nacional de Ciencia y Tecnología, México número 164725. Irma Reyes Jaramillo facilitó el uso del laboratorio para el análisis de suelo. Blanca Pérez-García permitió el uso del laboratorio de gametofitos para el cultivo de éstos. Robbin C. Moran hizo la revisión y sugerencias al manuscrito. Eulalio Águila Romero y Eduardo Águila Rodríguez ayudaron a la primera autora durante su trabajo de campo.

\section{RESUMEN}

Se estudiaron los patrones de distribución de 26 especies de pteridofitas como posibles indicadoras de alteración ambiental en los bosques templados de San Jerónimo Amanalco, Texcoco, Estado de México. La presencia y abundancia de la pteridoflora se relacionó con variables edáficas, topográficas y de vegetación en 100 sitios de muestreo en un área de 494 ha. Con base en el Análisis Canónico de Correspondencia se pudo explorar la relación entre variables. Se determinaron cinco paisajes en el área de estudio con base al grado de deterioro: erosión severa, erosión, montaña con deterioro moderado reversible, montaña sin deterioro aparente y cañada sin deterioro aparente. Cheilanthes bonariensis y Pellaea ternifolia son indicadores de deterioro ambiental. Los táxones que únicamente crecen en paisajes sin alteración aparente son: Adiantum andicola, Adiantum poiretii, Argyrochosma incana, Asplenium blepharophorum, Dryopteris pseudo filix-mas, Equisetum hyemale y Pteris cretica

Palabras clave: Pteridophyta, helechos, México, bosque templado, alteración ambiental.

\section{REFERENCIAS}

Barrington, D.S. 1993. Ecological and historical factors in fern biogeography. J. Biogeogr. 20: 275-280.
Bennett, D.P. \& D.A. Humphries. 1981. Introducción a la ecología de campo. H. Blume, Madrid, España.

Cosío Ruiz, C. 2001. Panorama socioeconómico y demográfico de Texcoco. Indicadores para la planeación municipal. Universidad Autónoma de Chapingo, Ciudad de México, México.

Gauch, H.G. 1982. Multivariate analysis in community ecology. Cambridge University, Cambridge, Reino Unido.

Instituto Nacional de Estadística, Geografía e Informática. 2000. Cuaderno Estadístico Municipal de Texcoco, INEGI, Estado de México, México.

Kovach, W.L. 2003. MVSP-A multivariate Statistical Package for Windows, ver. 3.2. Kovach Computing Services, Pentraeth, Gales, Reino Unido.

Krebs, C.J. 1985. Ecología: Estudio de la distribución y la abundancia. Harla, México D.F, México.

Landa, R., J. Meave \& J. Carabias. 1997. Environmental deterioration in rural Mexico: an examination of the concept. Ecolog. Appl. 7: 316-329.

Lande, R. 1996. Statistics and partition of species diversity, and similarity among multiple communities. Oikos 76: 5-13.

Lorea, F. \& R. Riba. 1990. Guía para la recolección y preparación de ejemplares para herbario de pteridofitas. Consejo Nacional de la Flora de México, México D.F., México.

Mickel, J.T. \& J.M. Beitel. 1988. Pteridophyte Flora of Oaxaca, Mexico. Mem. New York Bot. Gard. 46:1-568.

Mickel, J.T. \& A.R. Smith. 2004. The Pteridophytes of Mexico. Mem. New York Bot. Gard. 88: 1-1054.

Moran, R.C. \& R. Riba (eds.). 1995. Flora Mesoamericana. Psilotaceae a Salviniaceae. UNAM, México D.F., México.

Pérez-García, B., R. Riba \& I. Reyes Jaramillo. 1995. Helechos mexicanos: formas de crecimiento, hábitat y variantes edáficas. ContactoS 11: 22-27.

Reyes Jaramillo, I. 1996. Fundamentos teórico-prácticos de temas selectos de la ciencia del suelo. Parte I. Universidad Autónoma Metropolitana, México D.F., México.

Riba, R. 1994. XVII. El desarrollo de los estudios sobre pteridofitas de México, p. 333-341 In J. Llorente \& J. Luna (eds.). Taxonomía Biológica. Universidad 
Autónoma de México, Fondo de Cultura Económica, México D.F., México.

Riba, R. 1998. Pteridofitas mexicanas: distribución y endemismo, p. 369-384. In T.P. Ramamoorthy, R. Bye, A. Lot \& J. Fa (eds.). Diversidad biológica de México: orígenes y distribución. Instituto de Biología, UNAM, México D.F., México.

Rzedowski, J. 1978. Vegetación de México. Limusa, México D.F., México.

Smith, A.R. 1981. Pteridophytes, p. 1-370. In D.E. Breedlove (ed.). Flora of Chiapas. California Acad. Sci., San Francisco, California, EEUU.

Strasburger, E., F. Noll, H. Schenck \& A.F. Schimper. 1993. Tratado de botánica. Omega, Barcelona, España.
Tejero Díez, D. \& M.L. Arreguín Sánchez. 2004. Lista con anotaciones de las Pteridofitas del Estado de México. Acta Botán. Mex. 69: 1-82.

Tryon, R. 1986. The biogeography of species, with special reference to ferns. Bot. Rev. 52: 118-156.

Zimmermann, W. 1976. Evolución vegetal. Omega, Barcelona, España.

\section{REFERENCIA DE INTERNET}

Escalona Maurice, M.J. 2004. Análisis de las políticas públicas en la agricultura urbana caso Texcoco, México. (Consultado: 9 de septiembre del 2004, www.ipes.org/aguila/publicaciones/resumen $\% 20$ texcoco.htm). 